\title{
Two-line geometric derivation of the time derivatives of unit vectors in orthogonal
} curvilinear coordinate systems

\section{Gordon, R A}

Published in:

American Journal of Physics

Link to article, DOI:

10.1119/1.16627

Publication date:

1991

Document Version

Publisher's PDF, also known as Version of record

Link back to DTU Orbit

Citation (APA):

Gordon, R. A. (1991). Two-line geometric derivation of the time derivatives of unit vectors in orthogonal curvilinear coordinate systems. American Journal of Physics, 59(12), 1146-1147.

https://doi.org/10.1119/1.16627

\section{General rights}

Copyright and moral rights for the publications made accessible in the public portal are retained by the authors and/or other copyright owners and it is a condition of accessing publications that users recognise and abide by the legal requirements associated with these rights.

- Users may download and print one copy of any publication from the public portal for the purpose of private study or research.

- You may not further distribute the material or use it for any profit-making activity or commercial gain

- You may freely distribute the URL identifying the publication in the public portal

If you believe that this document breaches copyright please contact us providing details, and we will remove access to the work immediately and investigate your claim. 
$A=238$

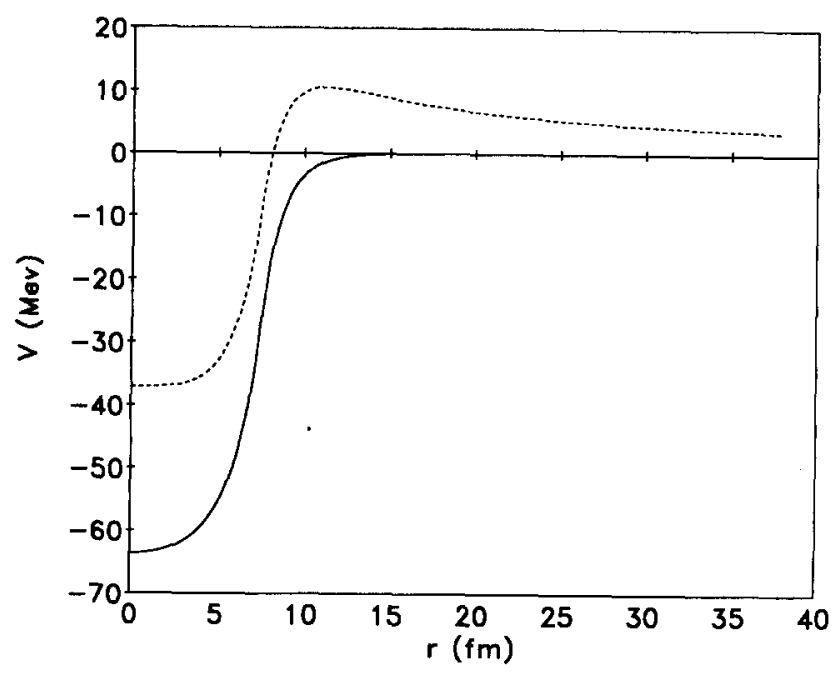

Fig. 2. $\bar{V}$ versus $r$ for a uranium nucleus $(A=238, Z=92)$, using $g^{2}=0.1 \hbar c$. Solid curve: $z=0$; dashed curve: $z=1$.

expected to be well described by the present spherically symmetric continuum model.

According to Eq. (3), the radii of the ${ }^{40} \mathrm{Ca}$ and ${ }^{238} \mathrm{U}$ nuclei are 4.17 and $7.56 \mathrm{fm}$, respectively. But it can be observed from Fig. 2, for example, that $\bar{V}$ reaches its maximum value beyond the nuclear surface because the nuclear force used here has a finite range $\lambda$. The position of the maximum can be obtained simply if one wishes to compute it.

\section{CONCLUDING REMARKS}

The Yukawa model has been used to calculate the net potential of a nucleus. The specific cases presented in Figs. 1 and 2 indicate that the Coulomb term becomes important in heavy nuclei, as expected. The resulting picture of the net nuclear potential is significantly different from the square well plus Coulomb tail approach that is often given in textbooks. ${ }^{6,7}$ Yet it is consistent with the conventional Woods-Saxon shapes which are often used in the research literature. ${ }^{8}$ Finally, note that the maximum of the nuclearCoulomb potential occurs at values of $r$ that are larger than the nuclear radius in heavy nuclear. This is because the nuclear force does not fall off to zero immediately at $r=R$, as assumed in simple square-well models.

' N. Gauthier, "Yukawa potential approach to the nuclear binding energy formula," Am. J. Phys. 58, 375-378 (1990).

${ }^{2}$ K. S. Krane, Introductory Nuclear Physics (Wiley, New York, 1987).

${ }^{3}$ H. A. Enge, Introduction to Nuclear Physics (Addison-Wesley, Reading, MA, 1966).

${ }^{4} \mathrm{~N}$. Gauthier, "Deriving a formula for nuclear radii using the measured atomic masses of elements," Am. J. Phys. 57, 344-346 (1989).

${ }^{5}$ L. R. B. Elton, Introductory Nuclear Theory (Saunders, Philadelphia, 1966), 2nd ed.

${ }^{\circ}$ B. L. Cohen, Concepts of Nuclear Physics (McGraw-Hill, New York, 1971), p. 259, Fig. 10-4.

${ }^{7}$ I. E. McCarthy, Introduction to Nuclear Theory (Wiley, New York, 1968).

${ }^{8}$ R. C. Barrett and D. F. Jackson, Nuclear Sizes and Structure (Oxford U.P., Oxford, 1977).

\title{
Two-line geometric derivation of the time derivatives of unit vectors in orthogonal curvilinear coordinate systems
}

\author{
R. A. Gordon \\ Physics Laboratory I, The Technical University of Denmark, Lyngby DK-2800, Denmark
}

(Received 3 December 1990; accepted for publication 6 March 1991)

Koo and Liew ${ }^{1}$ have used matrix methods to calculate the time derivatives of unit vectors in orthogonal curvilinear coordinate systems as an alternative to the algebraic derivations presented in standard textbooks. ${ }^{2-5}$ We would like to point out that a simple geometric derivation of the same transformation equations exists which is fully equivalent to both matrix and algebraic derivations but which, in the author's experience, is more transparent and appealing to beginning physics students. Since this geometric derivation is not given in standard textbooks, ${ }^{6}$ a brief outline of the derivation will be presented here for the case of cylindrical polar and spherical polar coordinate systems along with a short discussion of possible extensions of the deriva- tion to other less common coordinate systems.

The possibility of a geometric derivation of the time derivatives of unit vectors is based on the fact that any linear change in the unit vectors, such as the time derivative, can always be expressed as a simple rotation ${ }^{7}$ of the unit vector triad in three-dimensional space since only the orientation (but not the amplitude) of the individual orthogonal unit vectors can change in time. ${ }^{8,9} \mathrm{~A}$ two-line derivation of the time derivative of the unit vectors follows immediately from a knowledge of the angular velocity associated with the rotation of the unit vector triad.

More specifically, for the case of cylindrical polar coordinates, any instantaneous change in the unit vector triad 

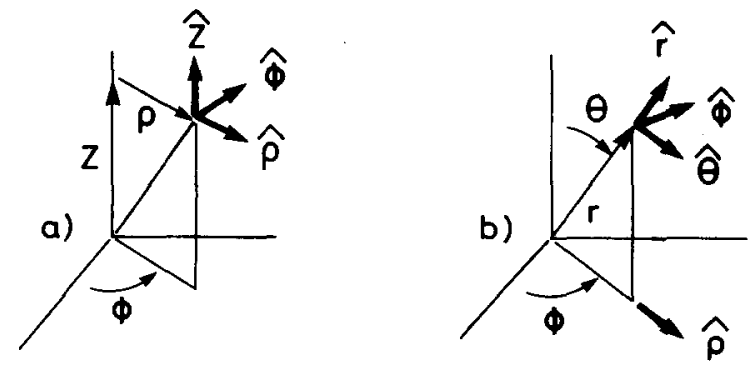

Fig. 1. (a) Here, $\rho, \phi$, and $z$ denote cylindrical polar coordinates and the corresponding unit vectors $\hat{\rho}, \hat{\phi}$, and $\hat{z}$, respectively. (b) Here, $r, \theta$ and $\phi$ denote spherical polar coordinates and the cooresponding unit vectors $\hat{r}$, $\hat{\theta}$, and $\hat{\phi}$, respectively. The cylindrical polar vector $\hat{\rho}$ lies in the same plane as $\hat{r}$ and $\hat{\theta}$.

$\hat{\rho}, \hat{\phi}, \hat{z}$ in time can be described in terms of a simple rotation of the unit vector triad about the $z$ axis at the angular velocity $\omega$ [see Fig. $1(\mathrm{a})]^{10}$ where

$$
\omega=\left(\frac{d \phi}{d t}\right) \hat{z}
$$

so that the time derivatives desired will be given immediately by:"

$$
\frac{d \hat{\rho}}{d t}=\omega \times \hat{\rho} ; \quad \frac{d \hat{\phi}}{d t}=\omega \times \hat{\phi} ; \quad \frac{d \hat{z}}{d t}=\omega \times \hat{z} .
$$

The well-known transformation equations for the time derivatives of cylindrical polar unit vectors then follow directly by straight forward inspection of the vector products in Eq. (2):

$$
\frac{d \hat{\rho}}{d t}=\left(\frac{d \phi}{d t}\right) \hat{\phi} ; \quad \frac{d \hat{\phi}}{d t}=-\left(\frac{d \phi}{d t}\right) \hat{\rho} ; \quad \frac{d \hat{z}}{d t}=0 .
$$

Analogously, for spherical polar coordinates, any instantaneous change in the unit vector triad $(\hat{r}, \hat{\theta}, \hat{\phi})$ in time can be represented [see Fig. $1(a)]^{12}$ as a simple rotation of the spherical polar unit vector triad about the $\hat{z}$ and $\hat{\phi}$ axes at the angular frequency $\omega$ given by

$$
\omega=\left(\frac{d \phi}{d t}\right) \hat{z}+\left(\frac{d \theta}{d t}\right) \hat{\phi}
$$

so that the time derivatives of spherical polar unit vectors will be given immediately by: ${ }^{11}$

$$
\frac{d \hat{r}}{d t}=\omega \times \hat{r} ; \quad \frac{d \hat{\theta}}{d t}=\omega \times \hat{\theta} ; \quad \frac{d \hat{\phi}}{d t}=\omega \times \hat{\phi} ;
$$

The standard transformation equations for the time derivatives of spherical polar unit vectors then follow directly by straightforward inspection of the vector products in Eq. (5):

$$
\begin{aligned}
& \frac{d \hat{r}}{d t}=\left(\frac{d \theta}{d t}\right) \hat{\theta}+\left(\frac{d \phi}{d t} \sin \theta\right) \hat{\phi}, \\
& \frac{d \hat{\theta}}{d t}=-\left(\frac{d \theta}{d t}\right) \hat{r}+\left(\frac{d \phi}{d t} \cos \theta\right) \hat{\phi},
\end{aligned}
$$

$$
\frac{d \hat{\phi}}{d t}=-\left(\frac{d \phi}{d t} \sin \theta\right) \hat{r}-\left(\frac{d \phi}{d t} \cos \theta\right) \hat{\theta}
$$

where we have used the fact that the unit vector $\hat{z}$ in Eq. (4) can by simple geometry [see Fig. 1(b)] be rewritten as

$$
\hat{z}=(\cos \theta) \hat{r}-(\sin \theta) \hat{\theta} .
$$

For other less well-known coordinate systems, it is not possible to write down the angular velocity associated with the rotation of the unit vector triad by simple inspection of the relevant orthogonal coordinate variables alone. Mathematical expressions for the angular velocity in terms of the relevant coordinate variables can be derived but such mathematical calculations greatly reduce the pedagogical advantage of a direct geometric derivation compared to standard algebraic or matrix derivations of the time derivatives. For such less common coordinate systems, the simplest geometric approach is probably that of Koo and Liew $^{1}$ where the rotation of the unit vector triad as a function of the coordinate variables is expressed in terms of cylindrical polar or spherical polar coordinate variables, $\phi$ or $\theta$, which are then substituted into the standard transformation equations [Eqs. (3) and (6)] already derived for cylindrical polar and spherical polar coordinate systems, respectively.

'W. K. Koo and Y. C. Liew, "Time derivatives of unit vectors in orthogonal curvilinear coordinate systems," Am. J. Phys. 57, 721-722 (1989). ${ }^{2}$ G. R. Fowles, Analytical Mechanics (Saunders, Tokyo, 1986), Chap. 1. ${ }^{3}$ D. Kleppner and R. J. Kolenkow, An Introduction to Mechanics (McGraw-Hill, London, 1973), Chap. 1.

${ }^{4}$ K. R. Symon, Mechanics (Addison-Wesley, London, 1971), Chap. 3.

${ }^{5} \mathrm{G}$. Christiansen, E. Both, and P. Sørensen, Mekanik (Stougaard Jensen, Copenhagen, 1989), Chap. 2.

${ }^{6}$ No claim to the originality of the derivation is made here and some teachers may employ similar geometric arguments in presenting the time derivatives of unit vectors. Combined algebraic-geometric derivations are also possible, e.g., see Ref. 4 .

${ }^{7}$ Trivial changes such as simple translations which leave both the orientation and amplitude of the unit vectors unchanged are ignored.

${ }^{8}$ W. K. Koo and Y. C. Liew, "A general method for differentiation of vectors in orthogonal systems of coordinates," Am. J. Phys. 57, 11181120 (1989).

${ }^{9}$ This equivalence to a rotation in three-dimensional space can also be seen from the general skew-symmetric form of the matrix transformation equations given by Koo and Liew in Ref. 1 or, more specifically, from Eqs. (3) and (6) in the text.

${ }^{10}$ Changes in the cylindrical polar variables $\rho$ and $z$ will not affect the orientation of the $(\hat{\rho}, \hat{\phi}, \hat{z})$ vector triad.

"Equations (2) and (5) for the time derivatives of a vector of constant amplitude rotation at the angular velocity $\omega$ are given in standard introductory texts (e.g., Refs. 2-5) and are readily derived and/or accepted by beginning physics students.

${ }^{12}$ Changes in the spherical polar variable $r$ will not affect the orientation of the $(\hat{r}, \hat{\theta}, \hat{\phi})$ vector triad. 